\title{
The impact of the rail welds geometry on the noise in urban zones
}

\author{
S. Lakusic \\ University of Zagreb, Faculty of Civil Engineering, Croatia
}

\begin{abstract}
During many years of supervision on construction and reconstruction of the tram tracks in Zagreb and Osijek (Croatia), it was noted that the damage caused by vehicles passing over uneven sections on the rail are large. Repair of the damage is expensive and in most of the cases requires suspension of the tram traffic and partially interferes with the road traffic as well, where the tram tracks are part of road construction. Existence of irregularity on rail running surface causes not only increased load on the track, but also reduces the effect of the rail fastening, deterioration of the track geometry, adverse impact on vehicles, reduce passengers' comfort and especially increases noise levels. In this paper, the impact of the irregularities in the zones of rail welds as a noise-contributing factor was studied. The measurements of the noise levels were performed in two phases. The first phase covered noise measurements for the tracks with irregularities in the welding zone. The geometry of the irregularities section was first recorded. The second phase covered measurements of noise levels at the same locations, but after the irregularities of the rail running surfaces were repaired. The measurements took into the account the tram types (seven types) and their speeds. That way it was possible to analyse the results to see to what extent the tram types and speeds do influence the increased noise levels. The analyses also took into the account the new low-floor tram type TMK 2200 produced in Zagreb and put into exploitation in June 2005. The research results helped to define the impact of the rails running surface geometry in the welding zone on the increased noise levels, and also to determine permissible tolerances for the geometry of welds on tram tracks in Zagreb.

Keywords: noise, tram type, rail weld, irregularity on rail running surface, smooth running surface.
\end{abstract}




\section{Introduction}

Reducing traffic noise in urban centres is today the focus of much attention. Were it not, there would be a danger that ambient noise levels would rise to unsatisfactory levels and therefore impact the standard of living. In order to undertake adequate measures to reduce noise levels, numerous scientists and experts worldwide are working through numerous projects today. Observing the noise caused by moving of a vehicle along rail track (trains, trams), the most of the attention is paid to researching the noise produced by contact of wheels and rails, and the noise caused by the vehicle itself. When the wheel and rail interaction is observed, the largest impact on increased noise level is caused by irregularities of the wheels (flat sections of the wheel, unround form of wheels), and irregularities of the rail surface (corrugations, rail joints, rail welds geometry). A tram line in Zagreb was selected for this study, due to its specificity in comparison with other cities in Europe which use the tram as the backbone of the public transportation system. The specificity is in the great traffic volume. Namely, some tram tracks in the centre of Zagreb have a traffic volume of up to 15 million gross tons a year per cross section with a tram service interval of less than 1 minute. For that reason in Zagreb was developed and is used a proprietary system for the permanent way of tram track construction, called 'Zagreb system'. The said system defines the means of fastening the rails to the base, and method for closing (paving) the track. This study addressed the influence of rail weld geometry on noise levels caused by moving of different types of trams vehicle along rail track. The weld geometry was investigated due to the fact that the contact between wheel-rail has a dominant role in the production of noise and vibration as the tram drives along the rail. Elevated noise levels resulting from tram traffic depend not only on irregularities on the rail running surface, but also on the tram vehicle itself (type, age, driving speed and type and condition of wheels). The study was conducted by the Faculty of Civil Engineering University of Zagreb in cooperation with Zagreb Streetcar Company, the company in charge of reconstruction and maintenance of the tracks in Zagreb.

\section{Description of field measurements}

The study of the impact of rail weld geometry was conducted on three streets in the City of Zagreb, Ilica Street (Location A), Maksimirska Street (Location B) and Savska Street (Location C). Ilica Street and Maksimirska Street represent the most important links between the western and eastern part of the city, until Savska Street represent the most important link between the north and south part of the city in terms of tram traffic. Reconstruction of the tram tracks was conducted in 1996 (Location A and Location B) and in 2002 (Location C). At all locations, tram tracks have been placed on continues concrete base. The fastening of the rails to the base was conducted with a direct elastic fastening system " $\mathrm{Zg} \mathrm{3/2"} \mathrm{on} \mathrm{Location} \mathrm{A} \mathrm{and} \mathrm{Location} \mathrm{B} \mathrm{and} \mathrm{with} \mathrm{an} \mathrm{indirect} \mathrm{double}$ elastic fastening system "DEPP" on Location C, fig. 1. The closure of the rails was carried out with reinforced concrete slabs on all locations, fig. 2. 
SYSTEM "DEPP"

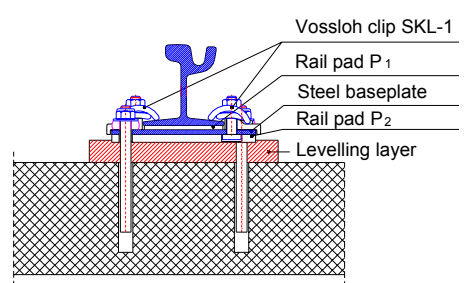

SYSTEM "Zg 3/2"

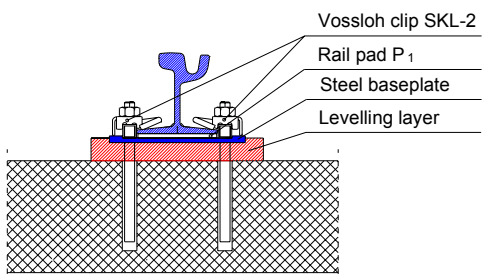

Figure 1: $\quad$ Fastening systems of tram tracks in Zagreb.

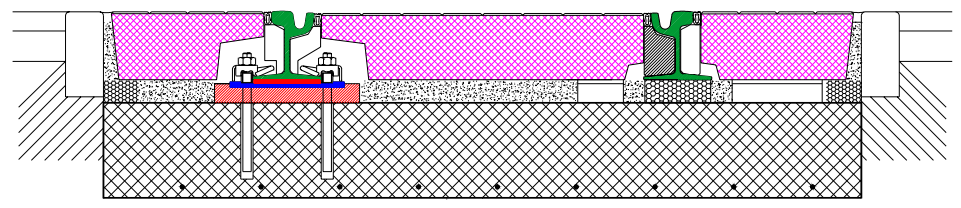

Figure 2: $\quad$ Cross section of tram track in Zagreb.

The traffic volume of the tram tracks at Location A totals 14 to 17 million gross tons per annum (MGT). Ilica Street is open to tram traffic and pedestrian traffic and for delivery vehicles in the morning hours, on the part near the centre of Zagreb. On other part, Ilica Street is open to both tram and vehicular traffic, primarily cars. A cross-section of Location A is shown in figure 3a. The traffic volume of the tram track in Maksimirska Street and Savska Street totals approximately 12 million gross tons per annum (MGT), according the data of Zagreb Streetcar Company, [1]. Unlike Location A, Maksimirska Street and Savska Street are subject to heavy tram traffic as well as a heavy traffic volume of road traffic (cars, trucks, buses). A higher tram speed than Location A, are also present here. Maksimirska Street and Savska Street are four lane roads, two lanes for each direction. The tram tracks are situated on the sides of the road and are an integral part of the road lanes. A cross section is shown in figure $3 b$.

a)

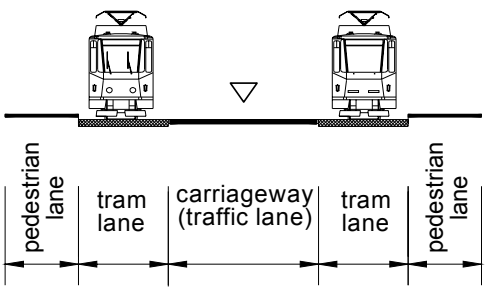

b)

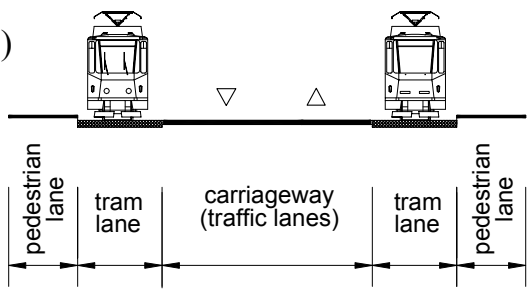

Figure 3: $\quad$ Cross section: a) Location A; b) Locations B and C.

In measuring noise levels, several types of tram vehicles according to different manufacturers were investigated: Type T4 (fig. 4) and Type KT4 (fig. 5) manufactured by CKD Tatra, Type TMK 101 (fig. 6) and Type TMK 201 (fig. 7) manufactured by Djuro Djakovic, Type GT6 (fig. 8) manufactured by Düwag and types TMK 2100 (fig. 9) and low floor tram TMK 2200 (fig. 10) 
646 Urban Transport XII: Urban Transport and the Environment in the 21st Century

manufactured by Koncar. These vehicles represent about $90 \%$ of the Zagreb Streetcar Company's rolling stock.

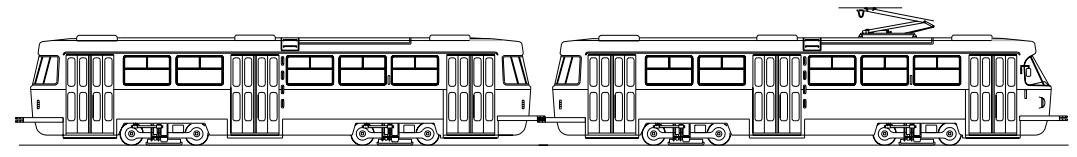

Figure 4: $\quad$ Tram type T4 (CKD Tatra).

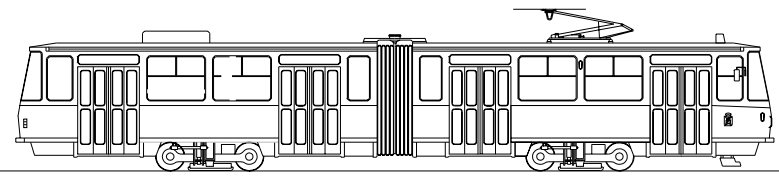

Figure 5: $\quad$ Tram type KT4 (CKD Tatra).

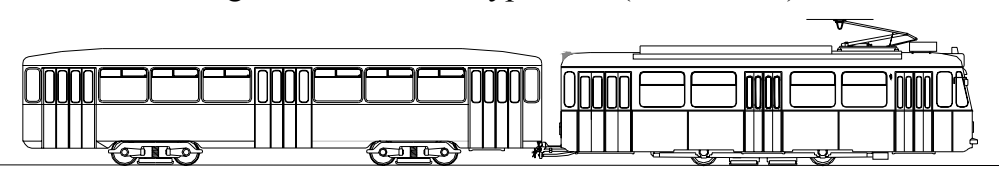

Figure 6: $\quad$ Tram type TMK 101 (Djuro Djakovic).

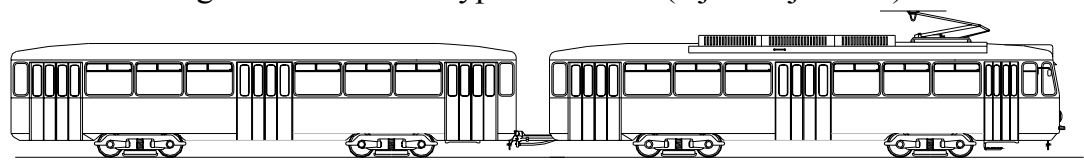

Figure 7: $\quad$ Tram type TMK 201 (Djuro Djakovic).

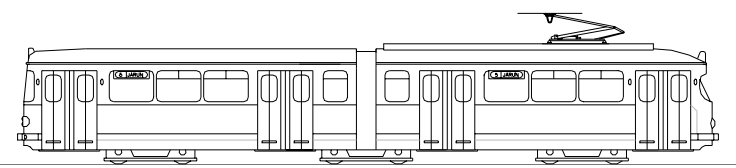

Figure 8: $\quad$ Tram type GT 6 (Düwag).

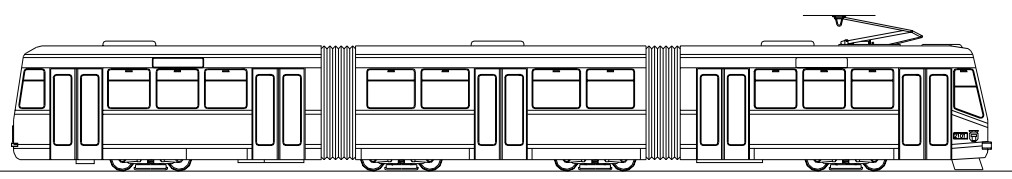

Figure 9: $\quad$ Tram type TMK 2100 (Koncar).

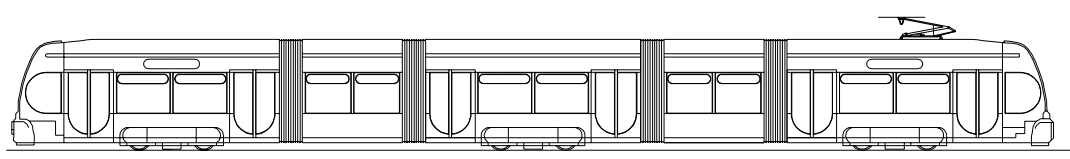

Figure 10: $\quad$ Tram type TMK 2200 (Koncar). 
The noise levels were measured with the following measuring equipment:

- Precision sound-meter, Bruel and Kjær 2238, serial number 2151881

- Microphone Bruel \& Kjær 4188, serial number 2141252

- Protection of microphone wind shield

- Aluminium telescopic stand for the sound-meter.

The software EVALUATOR 7820 by the same manufacturer was used for analysis of measurements. At each site, noise level was measured for a minimum of five passages of each type of tram vehicle. By measuring the interval of the passage of trams between two fixed points, tram driving speed was determined. Noise levels were measured one metre from the tram vehicles, at a height of 1.2 metres above the running surface of the rail (fig. 11). Noise level measurements were conducted in two phases: the first phase included noise measurements on tracks with irregularities at the welds, while the second phase included measurements of noise levels at the same locations following repairs to the irregularities of the rail running surfaces.

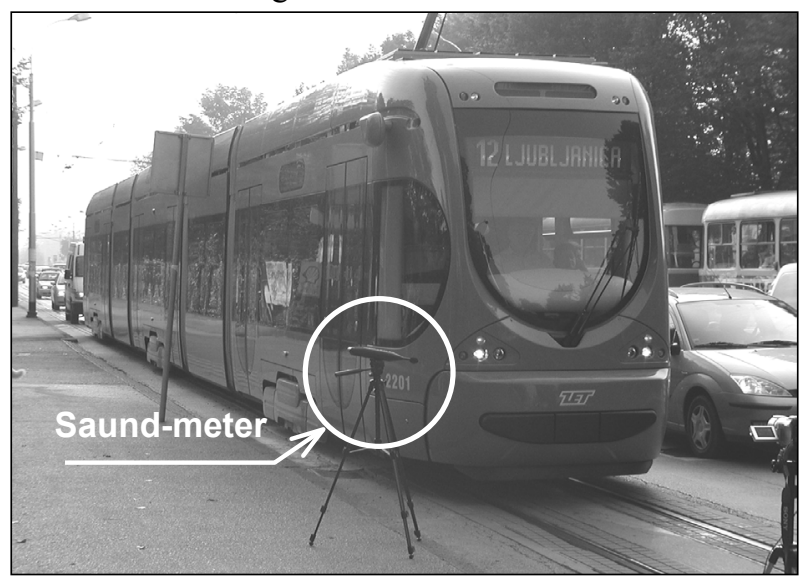

Figure 11: Position of instruments during measuring.

Prior to measuring noise levels, the geometry of the rail running surface at rail welds was measured. This measurement was conducted with a profilograph (device for measuring rail unevenness), fig. 12. This device has the capacity of measuring rail running surface irregularities at distances of 18,23 and $33 \mathrm{~mm}$ from the running edge of the rail. Considering that the subject of this study are tracks which have been in exploitation for 10 to 12 years, the measurement of unevenness was conducted at a distance of $23 \mathrm{~mm}$ from the rail edge, fig. 13 . The reason for selecting this measuring position is based on earlier studies conducted by the Faculty of Civil Engineering of the University of Zagreb, [2]. The differences between the uneven geometries measured at the same measurement location but from varying distances from the rail edge were analysed. It was established that for tracks which are in use for a certain period of time, the differences between recorded unevenness at $23 \mathrm{~mm}$ and $33 \mathrm{~mm}$ were virtually negligible. The positions of the measurement line of the geometry of the rail 
running surface in the weld zone (600 $\mathrm{mm}$ to the left and right of the weld) are seen in figure 13. The weld measurement scale was 1:10 in length and 30:1 vertically, while the instrument error is $0.05 \mathrm{~mm}$, [3]. Photographs of a weld on the Zagreb tram track and the recorded running surface are seen in fig. 13 (Location A) and fig. 14 (Location B).

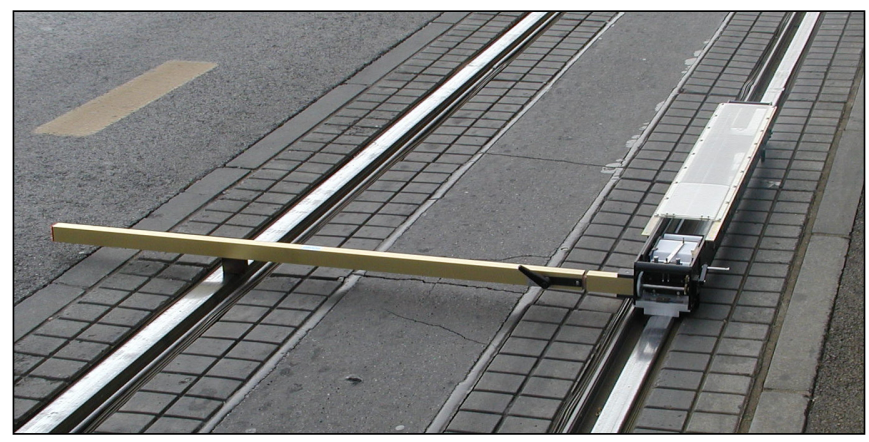

Figure 12: The profilograph - brand name CEMAFER.
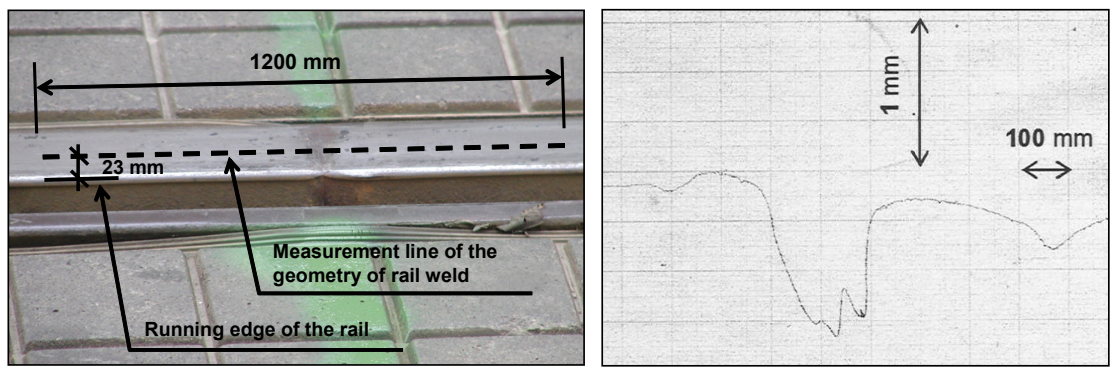

Figure 13: Picture of weld and vertical weld geometry - Location A.
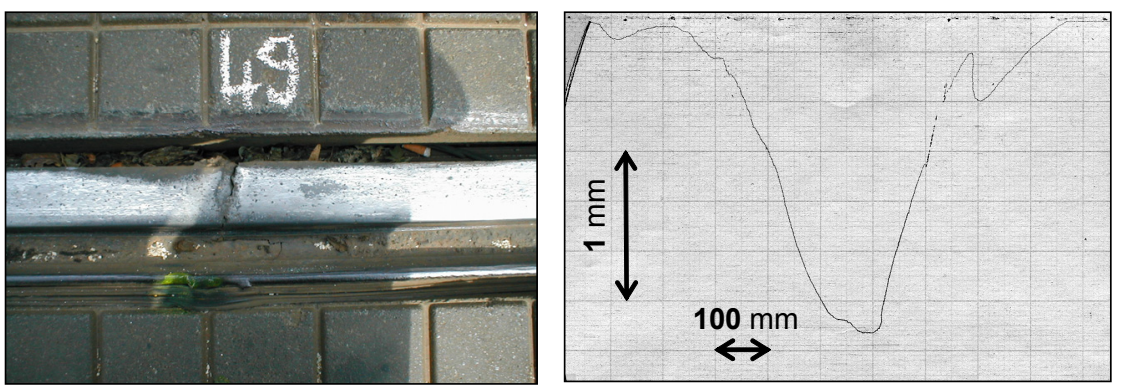

Figure 14: Photographs of weld and recorded weld geometry - Location B.

Following measurement of the unevenness geometry, an initial measurement of noise was conducted in the case with unevenness at the running surface and following repairs to the unevenness (fig. 15), noise level measurements were repeated. 

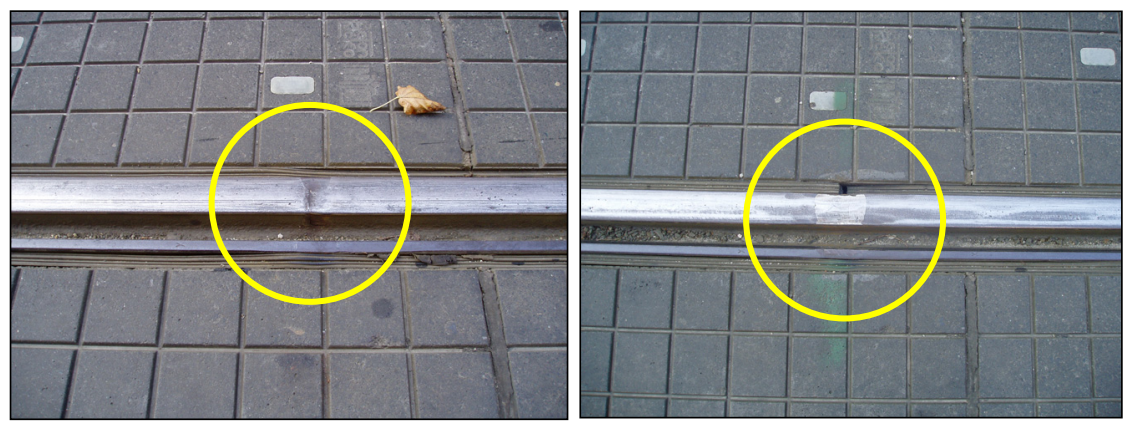

Figure 15: Unrepaired and repaired rail weld.

\section{Measurement results}

Noise levels were measured during tram passby, for a period of 20 to 40 seconds (depending on the tram driving speed). A characteristic depiction of the changes in noise levels over time are shown in fig. 16. The diagram shows the measurement noise levels at the time of passage of two tram vehicles over the irregularity in the rail weld. This diagram clearly shows the passage of each axle of the vehicle over the rail surface irregularity. The maximum noise levels (peaks) appeared during the passage of tram vehicles. Where irregularities on the rail surface are smaller, noise levels during tram passage are also smaller. The results of measurements taken at Location A with the presence of irregularity in the rail surface and following repairs to the rail surface are shown on fig. 17.

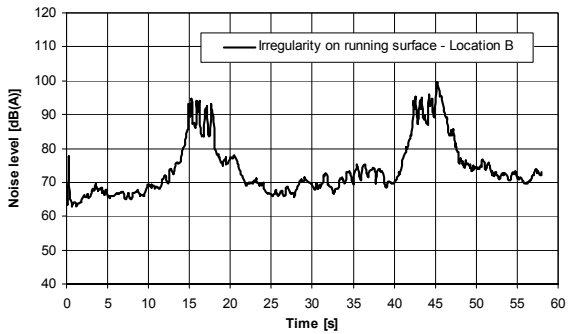

Figure 16: Characteristic depiction of change in noise level.

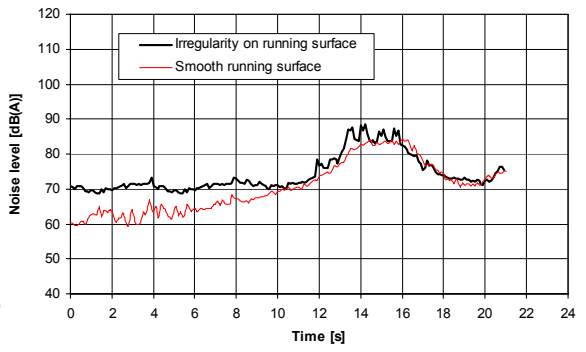

Figure 17: Noise levels prior to and following weld repairs.

The increased noise levels (peaks shown in figures) are clearly seen in the characteristic depiction of noise levels over time in figures 16 and 17. Following repairs to the irregularities, the sudden jumps in the noise level diagrams disappear and the maximum noise levels are thereby decreased (fig. 17).

To assess the impact of rail weld geometry on noise levels, peak noise levels were used. The reason for this lies in the fact that equivalent levels depend on the overall noise levels in the measurement period, and also contain noise from other traffic participants. 
a)

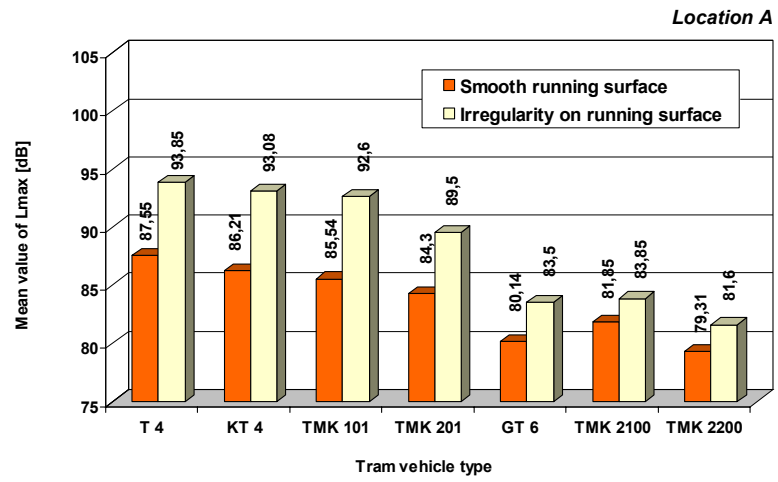

b)

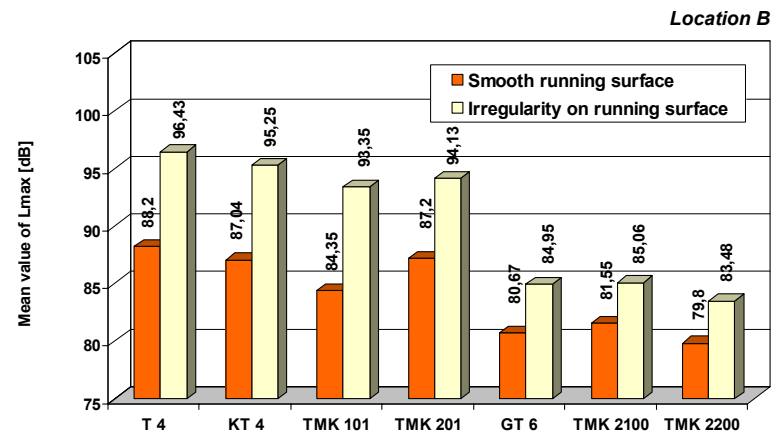

c)

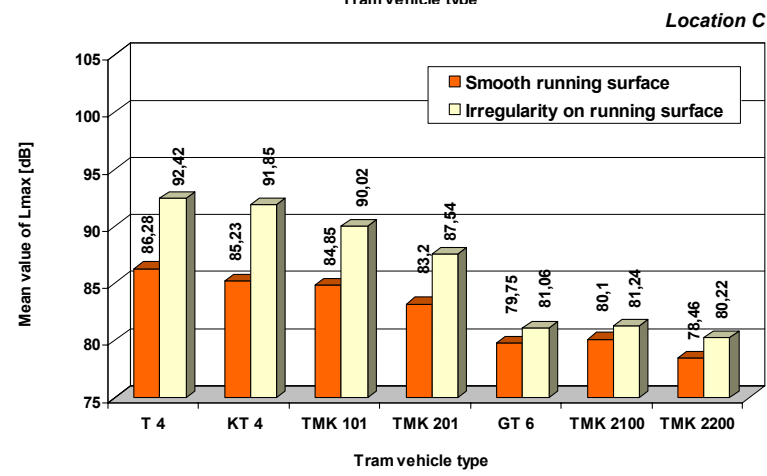

Figure 18: The maximal noise levels depending on tram and geometry of running surface.

In addition to comparing noise levels prior to and following repairs to weld irregularities, a comparison was also conducted of the mean values of noise level peaks dependent upon the type of tram. Tram vehicles which passed over the rails which were the subject of this study were investigated, as noted in point 2 . The mean values of noise level peaks dependent upon tram type is shown in figure 18a) for the measurement site at Location $\mathrm{A}$, in figure 18b) for the measurement site at Location $\mathrm{B}$ and in figure 18c) for the measurement site at Location $\mathrm{C}$. 
The mean value of maximal noise levels following repairs are 6 to $8 \mathrm{~dB}$ lower than measurements taken with the presence of rail surface irregularities (at the same location). Comparison of results of measurements prior to and following rail weld repairs at Locations A, B and C, it is clear that the level of noise at Location $C$ is about $5-10 \mathrm{~dB}$ less (prior to weld repairs) and about 3-6dB less (following weld repairs). The increased noise levels at Locations A and B are due partially to a greater tram speed and partially to greater geometric irregularities. At Location A, dents in the weld were $1.2 \mathrm{~mm}$ deep, while the same were $2.3 \mathrm{~mm}$ deep at Location B and $0.6 \mathrm{~mm}$ at Location C. The greater depth of irregularities is caused by the strong impact of the vehicle wheel at the moment the vehicle passes over the irregularity and results in a significant increase in noise levels. Comparison of the mean values for the maximal noise levels shows that the passage of the trams of the type T4, KT4, TMK 101 and TMK 201 causes larger increase of the noise levels (up to $7 \mathrm{~dB}$ ) as compared to the case where uneven driving surfaces exist. The trams of the GT6, TMK 2100 and TMK 2200 types had shown better results.

\section{Discussion}

Through the many years of supervision of reconstruction of the existing tram lines in Zagreb, it was noted that the damage incurred from the passage of vehicles over irregularities is great and that the repairs to damage are expensive and, for the most part, demand a cessation in tram traffic. This partially hinders the flow of road traffic at those locations where the tram tracks are part of the overall road construction, such as the case with the locations that were investigated in this study. It was also established that the greatest amount of damage to both the track construction and closure elements occur in zones of rail welds. Analysis of the damage which has occurred on tram rails in Zagreb shows that approximately $70 \%$ of damages occur at rail weld and composition sites [4, 5]. In the analysis of weld geometry on noise levels, it was found that in irregularities where the vertical deviation is greater than $0.33 \mathrm{~mm}$ (this listed vertical deviation was analysed at a length of $100 \mathrm{~mm}$, meaning that the maximum slope angle is $3.3 \mathrm{mrad}$ ), the noise level increased from 1.5 to $10 \mathrm{~dB}$ in comparison to the smooth running surface. In order to reduce increased noise levels during tram passby on tracks with rail running surface irregularities, it is proposed that the permitted deviation of the rail running surface geometry at weld locations have a maximum slope angle of $3.3 \mathrm{mrad}$.

\section{Conclusions}

Measuring noise levels was part of a broader project and is only shown in part in this paper, with the objective of comparing noise levels between tram passages on rail surfaces with and without irregularities. The presence of irregularities in the rail running surface causes not only significant damage to the rail, but also reduces the effect of the rail fastening, impairs the rail geometry, negatively impacts vehicles, reduces passenger comfort and results in an increase in noise 
levels. The result of measurements on rails with surface irregularities showed that noise level increases ranged from 3 to $10 \mathrm{~dB}$, dependent upon the size of the irregularity, type of tram vehicle and travelling speed. In order to reduce the noise levels stemming from rail running surface irregularities, it is necessary to conduct a control of the rail running surface geometry. The systematic monitoring of rail surface geometry, as a function of the passed tonnage, and the permanent repair of damaged spots (particularly at weld sites) can substantially improve passenger comfort and the life of the construction. On tracks with smooth rail running surfaces, not only is noise reduced during passage of tram vehicles, but there is also reduced load on the rails (reduced dynamic impacts), which thereby reduces the costs of rail maintenance. Also, measurements of the tram driving speed show that noise levels largely depend on the driving speed. Reductions in the driving speed of 10 to $15 \mathrm{~km} / \mathrm{h}$ reduced noise levels up to $5 \mathrm{~dB}$. The smallest increase of the noise levels caused by the tram traffic was for trams of the TMK 2200, TMK 2100 and GT6 types. The data for mean values of the maximal noise levels, shown on the figure 18, confirm this. The figure shows comparisons of the tram vehicle types regarding the noise levels generated by their traffic, depending also on the characteristics of the tram tracks (rail fastening system and the geometry of the running surfaces). Better results for the tram types TMK 2200, TMK 2100 and GT6, as far as noise generation is concerned can be explained that these vehicle types have wheels with elastic elements and a different concept for the relations between the bogie and the tram body. The largest increase in the noise level is caused by traffic of T4 and KT4 tram types, manufactured by CKD Tatra and TMK 101 and TMK 201 types (manufactured by Đuro Đakovic). These vehicles are between 25 to 35 years old, their concept is out of date, and it cannot be neither advised, nor justified to propose their general reparation. For that reason, the Zagreb Streetcar Company contracted manufacture of 70 new low-floor trams from Croatian consortium CROTRAM, formed by companies Koncar and Gredelj. The analysis of the results of measuring the impact of the TMK 2200 tram type on the noise levels has shown that, compared to the other vehicles in the Zagreb Streetcar Company rolling stock, this tram type shows the best characteristics.

\section{References}

[1] Measured traffic volume, Zagreb Streetcar Company: Zagreb, 2000.

[2] Lakusic, S., Dynamic behaviour of the tram-track interaction, Dissertation, Faculty of Civil Engineering: Zagreb, 2003.

[3] Fastenrath, F., (ed). Die Eisennbahnschiene, Verlag: Berlin, 1977.

[4] Lakušić S., Rak M., Experimental analysis of the rail behaviour in the case with irregularity on running surface and without it, Proc. Of the $20^{\text {th }}$ Danubia-Adria Symposium, eds. F. Thamm \& L. Borbas, Hungarian Scientific Society of Mechanical Engineering: Gyor, pp. 194-196, 2003.

[5] Esveld, C., Modern Railway Track, Second edition, TU-Delft, 2001. 